\title{
Severe Acute Respiratory Syndrome Coronavirus 2 Infection among Returnees to Japan from Wuhan, China, 2020
}

\author{
Yuzo Arima, ${ }^{1}$ Satoshi Kutsuna, ${ }^{1}$ Tomoe Shimada, Motoi Suzuki, Tadaki Suzuki, Yusuke Kobayashi, \\ Yuuki Tsuchihashi, Haruna Nakamura, Kaoru Matsumoto, Asuka Takeda, Keisuke Kadokura, Tetsuro Sato, \\ Yuichiro Yahata, Noriko Nakajima, Minoru Tobiume, Ikuyo Takayama, Tsutomu Kageyama, Shinji Saito, \\ Naganori Nao, Tamano Matsui, Tomimasa Sunagawa, Hideki Hasegawa, Kayoko Hayakawa, \\ Shinya Tsuzuki, Yusuke Asai, Tetsuya Suzuki, Satoshi Ide, Keiji Nakamura, Yuki Moriyama, Noriko Kinoshita, \\ Yutaro Akiyama, Yusuke Miyazato, Hidetoshi Nomoto, Takato Nakamoto, Masayuki Ota, Sho Saito, \\ Masahiro Ishikane, Shinichiro Morioka, Kei Yamamoto, Mugen Ujiie, Mari Terada, Haruhito Sugiyama, \\ Norihiro Kokudo, Norio Ohmagari, Makoto Ohnishi, Takaji Wakita, the COVID-19 Response Team
}

In early 2020, Japan repatriated 566 nationals from China. Universal laboratory testing and 14-day monitoring of returnees detected 12 cases of severe acute respiratory syndrome coronavirus 2 infection; initial screening results were negative for 5 . Common outcomes were remaining asymptomatic $(n=4)$ and pneumonia $(n=6)$. Overall, screening performed poorly.

W ith the emergence of severe acute respiratory syndrome coronavirus 2 (SARS-CoV-2) in $\mathrm{Wu}$ han, China, several countries, including Japan, repatriated their nationals (1-3). During January 29-31, 2020, a total of 566 Japanese nationals were repatriated via 3 chartered flights from Wuhan (206, 210, and 150 passengers). After passengers disembarked in Tokyo, Japan, quarantine officials assessed them for signs/symptoms (e.g., fever, respiratory illness) of coronavirus disease (COVID-19) (4). A total of

Author affiliations: National Institute of Infectious Diseases, Tokyo, Japan (Y. Arima, T. Shimada, M. Suzuki, T. Suzuki, Y. Kobayashi, Y. Tsuchihashi, H. Nakamura, K. Matsumoto, A. Takeda, K. Kadokura, T. Sato, Y. Yahata, N. Nakajima, M. Tobiume, I. Takayama, T. Kageyama, S. Saito, N. Nao, T. Matsui, T. Sunagawa, H. Hasegawa, M. Ohnishi, T. Wakita); National Center for Global Health and Medicine, Tokyo (S. Kutsuna, K. Hayakawa, S. Tsuzuki, Y. Asai, T. Suzuki, S. Ide, K. Nakamura, Y. Moriyama, N. Kinoshita, Y. Akiyama, Y. Miyazato, H. Nomoto, T. Nakamoto, M. Ota, S. Saito, M. Ishikane, S. Morioka, K. Yamamoto, M. Ujiie, M. Terada, H. Sugiyama, N. Kokudo, N. Ohmagari)

DOI: https://doi.org/10.3201/eid2607.200994
28 symptomatic passengers were transferred to select hospitals for isolation. The remaining 538 were transported to a designated hospital, where another 35 were found to be symptomatic and were hospitalized there or transferred to other hospitals, leaving 503 asymptomatic persons for observation in quarantine (Figure).

\section{The Study}

We conducted day 1 entry screening by testing oropharyngeal swab samples collected from all 566 returnees at the hospitals to which they were initially transported for SARS-CoV-2 (4); all tests were based on the real-time reverse transcription PCR developed by the National Institute of Infectious Diseases (5). Hospitalized patients in isolation and asymptomatic returnees in quarantine were monitored daily for 14 days. If any signs/symptoms developed in a quarantined person, that person was transported to a designated hospital and oropharyngeal swab samples were collected for testing. We conducted exit screening for quarantined persons who remained illness-free by collecting oropharyngeal swab samples on day 14 . The National Institute of Infectious Diseases Ethics Committee approved the study (registration no. 1096), and all 566 returnees who provided specimens gave written informed consent.

Among the 63 passengers who were symptomatic at entry screening, 2 (3.2\%) were positive by PCR

\footnotetext{
${ }^{1}$ These authors contributed equally to this article.
} 
(Figure); test results were subsequently positive for 2 more. For 1 of these patients, pneumonia was diagnosed on day 1 and a sputum sample was positive on day 3; the other patient had fever and cough on day 1 , pneumonia diagnosed on day 2 , and a positive oropharyngeal swab sample on day 6. Excluding 1 patient who remained hospitalized for stroke, the remaining 58 patients were transferred to designated quarantine facilities after confirmation of good health and negative PCR results; all 58 remained asymptomatic after discharge, and PCR results were negative at exit screening.

For the 503 asymptomatic/subclinical passengers, entry-screening PCR results were positive for 5 $(1.0 \%)$ (Figure); 3 remained asymptomatic, but mild signs/symptoms (fever, headache, sore throat) developed for 2 persons ( 1 on day 2,1 on day 4 ). Of the remaining 498 persons with negative PCR results, 484 were quarantined at designated facilities and 14 at home. During quarantine, fever developed in 1 facility-quarantined and 1 home-quarantined person on day 10; both were confirmed positive by PCR, and pneumonia subsequently developed in both. The facility-quarantined case-patient was in a single room; no other person from this facility acquired COVID-19 or had a positive test result at exit screening. One person who remained asymptomatic had a positive test result at exit screening. Exit-screening results are pending for the patient hospitalized for stroke and the remaining 13 homequarantined persons.

Among the 566 returnees, 12 cases of SARS-CoV-2 infection were detected; 540/541 facility-quarantined persons were confirmed negative by PCR performed on days 1 and 14 (197/197, 199/199, and 144/145 for the 3 flights). Entry screening detected 7 infections, for an infection point prevalence of $1.2 \%$; infection period prevalence was $2.2 \%$ (12/552 returnees with complete follow-up). Despite universal testing, entry screening captured only $7 / 12$ cases ( $58.3 \%$ sensitivity).

Entry screening, day $1 \quad$ 14-day monitoring $\quad$ Exit screening, day 14

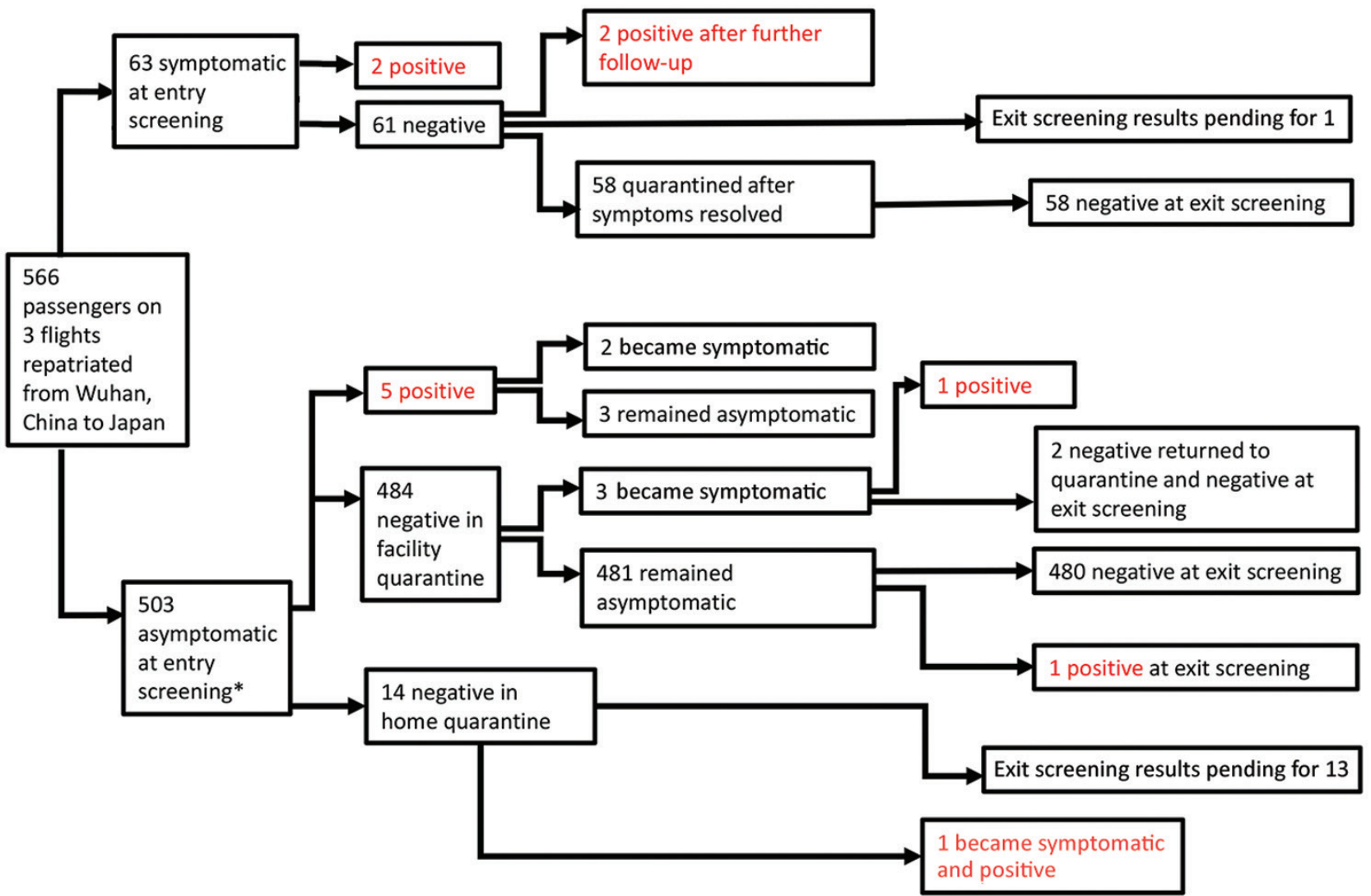

Figure. Results of testing 566 Japanese returnees from Wuhan, China, for severe acute respiratory syndrome coronavirus 2 by real-time reverse transcription PCR, January-February 2020. *Two persons were sampled on day 3, when they provided informed consent. 
Table. Distribution of severe acute respiratory syndrome coronoavirus 2 infections and clinical outcomes, by age group, among 566 Japanese returnees from Wuhan, China, January-February 2020

\begin{tabular}{|c|c|c|c|c|c|c|}
\hline \multirow[b]{2}{*}{ Age group, y } & \multirow[b]{2}{*}{ No. returnees* } & \multirow[b]{2}{*}{ No. (\%) infected $\dagger$} & \multicolumn{4}{|c|}{ Outcome } \\
\hline & & & No. asymptomatic & $\begin{array}{l}\text { No. with } \\
\text { mild illness }\end{array}$ & $\begin{array}{c}\text { No. with } \\
\text { pneumonia }\end{array}$ & No. deaths \\
\hline$<10$ & 6 & 0 & 0 & 0 & 0 & 0 \\
\hline $10-19$ & 4 & 0 & 0 & 0 & 0 & 0 \\
\hline $20-29$ & 90 & 0 & 0 & 0 & 0 & 0 \\
\hline $30-39$ & 138 & $2(1.4)$ & 2 & 0 & 0 & 0 \\
\hline $40-49$ & 168 & $4(2.4)$ & 0 & 1 & 3 & 0 \\
\hline $50-59$ & 119 & $5(4.2)$ & 1 & 1 & 3 & 0 \\
\hline $60-69$ & 26 & $1(3.8)$ & 1 & 0 & 0 & 0 \\
\hline $70-79$ & 1 & 0 & 0 & 0 & 0 & 0 \\
\hline
\end{tabular}

${ }^{*}$ Day-14 exit-screening test results pending for 14 persons.

†Testing by reverse transcription PCR.

Although screening symptomatic passengers (3.2\%) was more efficient than screening all passengers $(1.2 \%)$, screening only symptomatic passengers missed 5/7 prevalent infections at entry. Among symptomatic passengers, with 2 initially negative persons subsequently testing positive, entry-screening sensitivity was $2 / 4(50 \%)$. Among asymptomatic passengers, with 3 initially negative persons subsequently testing positive, entry-screening sensitivity was $5 / 8(62.5 \%)$.

\section{Conclusions}

Testing all returnees-with follow-up for disease onset and course-enabled us to evaluate the spectrum of severity for SARS-CoV-2 infections (Table). From least to most severe, 4 patients experienced asymptomatic infection, 2 mild illness, and 6 pneumonia. Prospective monitoring proved essential because of the 7 prevalent infections at entry, 5 were asymptomatic, 1 mild, and 1 pneumonia. Even with potential underascertainment of asymptomatic cases because of a lack of serologic assessment $(6,7)$ (i.e., interval-censoring during screening tests), it is noteworthy that $4 / 12$ persons with infections were asymptomatic. Although numbers are small, severity seemed to be age dependent (Table). No infections were detected among the 100 persons $<30$ years of age; of the 2 infections detected among the 138 persons 30-39 years of age, both persons were asymptomatic. Although no person in this study died, only 1 was $>69$ years of age. Regarding sex, excluding 1 returnee for whom sex was unknown and 14 for whom exit-screening results are pending, of the remaining 551 returnees, 9 (1.8\%) of the 506 male passengers ( 2 asymptomatic, 2 mild, 5 pneumonia) and $3(6.7 \%)$ of the 45 female passengers (2 asymptomatic, 1 pneumonia) were infected.

Our findings have public health implications. As recently reported (1), we found that symptom-based screening performed poorly, missing asymptom- atic and presymptomatic cases. Even with universal screening, nearly half of cases were missed. Because an asymptomatic case was detected at exit screening, limiting testing of quarantined persons to those with signs/symptoms would have missed such a case; with exit-screening results pending for 14 returnees, sensitivity could be lower. The poor sensitivity of single-point testing highlights the challenges of detecting SARS-CoV-2 infections.

The potentially long incubation period of COVID-19 was consistent with that recently reported $(8,9)$ and contributed to the large proportion of missed cases. Active daily monitoring ensured that specific illness-onset times were captured, protected from the limitations associated with patient recall of symptom onset (10). Although exposure to SARSCoV-2 occurred at some time before quarantine (i.e., left-censore d), our setting enabled us to estimate the minimum incubation period for each incident symptomatic case by taking the return date as the exposure time. Determining the specific exposure time can be difficult and is conditional according to the definition of contact. Given such qualifications, a conservative minimum incubation period of 10 days obtained prospectively in a clean quarantine setting, without recall or assumptions regarding transmission modes, is noteworthy.

Testing and follow-up of all returnees provided valuable information about the spectrum of SARSCoV-2 infection. Most reported data have been from medically attended patients, skewed toward symptomatic patients and more severe cases, limiting our knowledge of the clinical spectrum of infection $(6,11)$. In our setting, we could remove the influence of patients' health-seeking behaviors and clinicians' diagnostic practices and found that $4 / 12$ case-patients were asymptomatic. At the same time, of the 8 case-patients who experienced symptoms, pneumonia developed in 6 . Our findings were also consistent with the reported age-dependent nature 
of COVID-19 (2,12-14); infection and clinical attack rates were lower among younger persons. Shedding light on the severity pyramid among those infected - not only among those who sought careprovides an evidence base for risk communication, healthcare planning, and public health response. Combined with reports suggesting transmissibility of SARS-CoV-2 from asymptomatic/subclinical case-patients $(1,10,15,16)$, our findings suggest that controlling COVID-19 through the usual tools of syndrome-based surveillance and contact tracing alone may be difficult.

When confronted with an emerging pathogen, researchers can generate critical epidemiologic information by studying quarantined populations. As with the First Few X study (7), our design is protected from the usual biases of passively reported surveillance data. Aggregating high-quality data from these types of investigations can build a larger severity pyramid, enabling reliable estimation of various severity measures (e.g., symptomatic proportion of infected case-patients, case severity proportion among those who are symptomatic). We recommend using similar assessments to help elucidate the epidemiology of SARS-CoV-2 and inform public health response.

Members of the COVID-19 response team: Akira Ainai, Minetaro Arita, Tomoko Arita, Hideki Asanuma, Seiichiro Fujisaki, Itsuki Hamamoto, Yuichi Harada, Shinichiro Hirai, Shun Iida, Yoshiyuki Ishii, Shigeyuki Itamura, Naoko Iwata-Yoshikawa, Takayuki Kanno, Harutaka Katano, Fumihiro Kato, Hirofumi Kato, Hiroshi Katoh, Minoru Kidokoro, Noriko Kishida, Osamu Kotani, Toru Kubota, Iwao Kukimoto, Madoka Kuramitsu, Tomoko Kuwahara, Shutoku Matsuyama, Seiichiro Mori, Yoshio Mori, Koichi Murakami, Tsutomu Murakami, Noriyo Nagata, Seishiro Naito, Kazuya Nakamura, Yuichiro Nakatsu, Mina Nakauchi, Eri Nobusawa, Kiyoko Okamoto, Kazu Okuma, Noriyuki Otsuki, Masumichi Saito, Koji Sakai, Kouji Sakai, Masafumi Sakata, Kaori Sano, Kayoko Sato, Yuko Sato, Fumio Seki, Noriko Shimasaki, Hiroyuki Shimizu, Masayuki Shirakura, Kazuya Shirato, Kenji Someya, Ryosuke Suzuki, Yasushi Suzuki, Maino Tahara, Hitoshi Takahashi, Kenta Takahashi, Emi Takashita, Makoto Takeda, Takamasa Takeuchi, Michiko Tanaka, Kenta Tezuka, Kenzo Tokunaga, Yuji Wada, Kana Watanabe, Shinji Watanabe, Aya Zamoto-Niikura, (National Institute of Infectious Diseases); Tomoko Date (National Center for Global Health and Medicine); Takashi Chiba, Mami Nagashima, Kenji Sadamasu, Kazuhisa Yoshimura (Tokyo Metropolitan Institute of Public Health); Manami Yanagawa (Ministry of Health, Labour and Welfare).

\section{Acknowledgments}

We acknowledge the dedicated work of the frontline clinicians and the staff at the quarantine facilities, the workers at the local public health centers and prefectural and municipal public health institutes, and the Japan Ministry of Health, Labour and Welfare.

We also thank the quarantined returnees for their patience and participation.

This study was supported in part by a grant-in-aid from the Japan Agency for Medical Research and Development (grant nos. JP19fk0108104 and JP19fk0108110).

Dr. Arima is an affiliate researcher at the Infectious Disease Surveillance Center, National Institute of Infectious Diseases in Tokyo, Japan. His research interests include infectious disease epidemiology and surveillance in the context of public health practice.

\section{References}

1. Hoehl S, Rabenau H, Berger A, Kortenbusch M, Cinatl J, Bojkova D, et al. Evidence of SARS-CoV-2 infection in returning travelers from Wuhan, China. N Engl J Med. 2020;382:1278-80. https:/ / doi.org/10.1056/NEJMc2001899

2. Jernigan DB; CDC COVID-19 Response Team. Update: public health response to the coronavirus disease 2019 outbreak - United States, February 24, 2020. MMWR Morb Mortal Wkly Rep. 2020;69:216-9. https://doi.org/10.15585/ mmwr.mm6908e1

3. Ng OT, Marimuthu K, Chia PY, Koh V, Chiew CJ, De Wang L, et al. SARS-CoV-2 infection among travelers returning from Wuhan, China. N Engl J Med. 2020;382:14768. https:// doi.org/10.1056/NEJMc2003100

4. Kutsuna S, Suzuki T, Hayakawa K, Tsuzuki S, Asai Y, Suzuki T, et al. SARS-CoV-2 screening test for Japanese returnees 1 from Wuhan, China. 2 January 2020. Open Forum Infectious Diseases. In press 2020.

5. Shirato K, Nao N, Katano H, Takayama I, Saito S, Kato F, et al. Development of genetic diagnostic methods for novel coronavirus 2019 (nCoV-2019) in Japan. Jpn J Infect Dis. 2020 Feb 18 [Epub ahead of print]. https:/ / doi.org/10.7883/ yoken.JJID.2020.061

6. Lipsitch M, Swerdlow DL, Finelli L. Defining the epidemiology of COVID-19-studies needed. N Engl J Med. 2020;382:1194-6. https://doi.org/10.1056/NEJMp2002125

7. World Health Organization. The First Few X (FFX) cases and contact investigation protocol for 2019-novel coronavirus (2019-nCoV) infection [cited 2020 Mar 23]. https:// www.who. int/publications-detail/the-first-few-x-(ffx)-cases-andcontact-investigation-protocol-for-2019-novel-coronavirus(2019-ncov)-infection

8. Morens DM, Daszak P, Taubenberger JK. Escaping Pandora's box - another novel coronavirus. N Engl J Med. 2020;382:1293-5. https:/ / doi.org/10.1056/NEJMp2002106

9. Backer JA, Klinkenberg D, Wallinga J. Incubation period of 2019 novel coronavirus (2019-nCoV) infections among travellers from Wuhan, China, 20-28 January 2020. Euro Surveill. 2020;25. https://doi.org/10.2807/1560-7917. ES.2020.25.5.2000062

10. Tong ZD, Tang A, Li KF, Li P, Wang HL, Yi JP, et al. Potential presymptomatic transmission of SARS-CoV-2, Zhejiang Province, China, 2020. Emerg Infect Dis. 2020;26:1050-2. https://doi.org/10.3201/eid2605.200198 
11. Munster VJ, Koopmans M, van Doremalen N, van Riel D, de Wit E. A novel coronavirus emerging in China-key questions for impact assessment. N Engl J Med. 2020;382:692-4. https://doi.org/10.1056/NEJMp2000929

12. World Health Organization. Report of the WHO-China Joint Mission on Coronavirus Disease 2019 (COVID-19) [cited 2020 Mar 23]. https://www.who.int/docs/default-source/coronaviruse/ who-china-joint-mission-on-covid-19-final-report.pdf

13. Li Q, Guan X, Wu P, Wang X, Zhou L, Tong Y, et al. Early transmission dynamics in Wuhan, China, of novel coronavirus-infected pneumonia. N Engl J Med. 2020;382:1199-207. https:/ / doi.org/10.1056/NEJMoa2001316

14. Guan WJ, Ni ZY, Hu Y, Liang WH, Ou CQ, He JX, et al.; China Medical Treatment Expert Group for COVID-19. Clinical characteristics of coronavirus disease 2019 in
China. N Engl J Med. 2020 Feb 28 [Epub ahead of print]. https://doi.org/10.1056/NEJMoa2002032

15. Zou L, Ruan F, Huang M, Liang L, Huang H, Hong Z, et al. SARS-CoV-2 viral load in upper respiratory specimens of infected patients. N Engl J Med. 2020;382:1177-9.

16. Rothe C, Schunk M, Sothmann P, Bretzel G, Froeschl G, Wallrauch C, et al. Transmission of 2019-nCoV infection from an asymptomatic contact in Germany. N Engl J Med. 2020;382:970-1.

Address for correspondence: Yuzo Arima, Infectious Disease Surveillance Center, National Institute of Infectious Diseases, 1-23-1 Toyama, Shinjuku-ku, Tokyo 162-8640, Japan; email arima@niid.go.jp

\section{Emerging Infectious Diseases Spotight Topics}
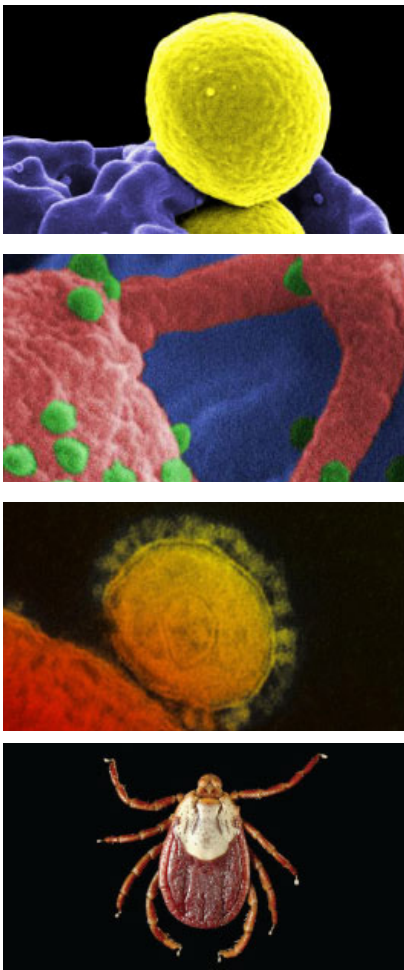
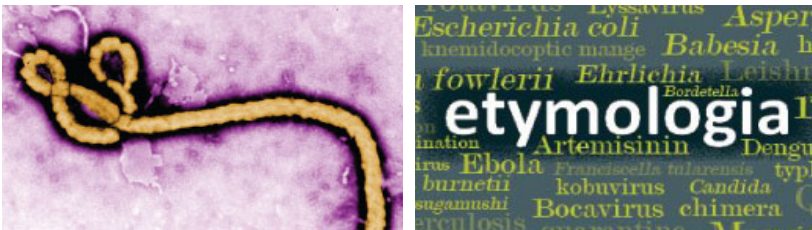

Antimicrobial resistance • Ebola Etymologia • Food safety • HIV-AIDS Influenza • Lyme disease • Malaria MERS • Pneumonia • Coronavirus Rabies • Tuberculosis • Ticks • Zika
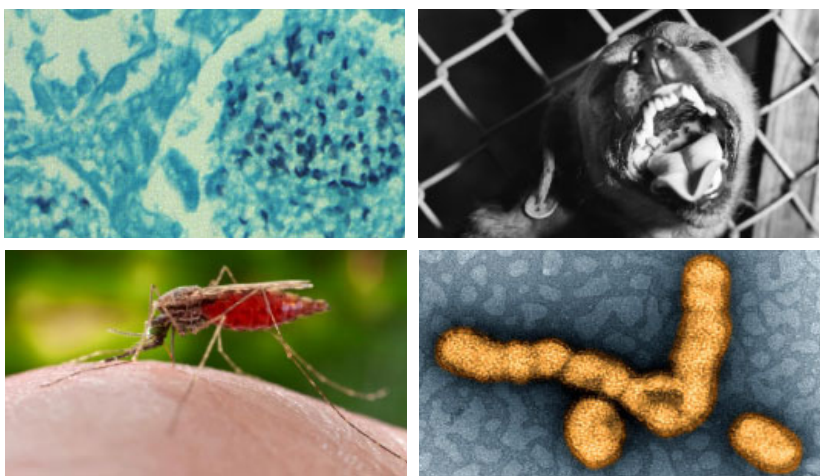
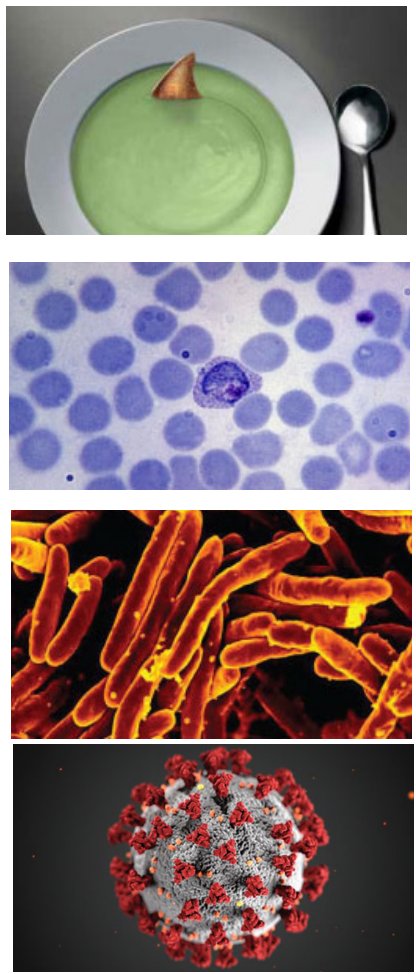

EID's spotlight topics highlight the latest articles and information on emerging infectious disease topics in our global community https://wwwnc.cdc.gov/eid/page/spotlight-topics 